\title{
Study of Linear Poly(p-chlorostyrene)-Diluent Systems. III. Unperturbed Chain Dimensions in Eleven Theta Solvents
}

\author{
Yoshinobu Izumi and Yasuhiro Miyake \\ Department of Polymer Science, Faculty of Science, \\ Hokkaido University, Sapporo, Japan.
}

(Received April 4, 1972)

\begin{abstract}
The unperturbed mean square end-to-end distance $\left\langle r_{0}^{2}\right\rangle$ and its temperature coefficient $\mathrm{d} \ln \left\langle r_{0}^{2}\right\rangle / \mathrm{d} T$ for linear poly ( $p$-chlorostyrene) have been studied by intrinsic viscosity measurements at the $\theta$ temperatures in the homologous theta solvents. Some of them are the solvents with LCST, i.e., isopropylacetate and $t$-butylacetate; ethylcarbitol and $n$-butylcarbitol, and others are the solvents with UCST, i.e., ethylbenzene and isopropylbenzene; carbon tetrachloride and tetrachloroethylene; methyl chloroacetate, ethyl chloroacetate and isopropyl chloroacetate. The average values of characteristic ratio $\left\langle r_{0}^{2}\right\rangle / n l^{2}$, steric factor $\sigma$ and temperature coefficient $\mathrm{d} \ln \left\langle r_{0}{ }^{2}\right\rangle / \mathrm{d} T$ evaluated by assuming constant $\Phi=2.87 \times 10^{21}$ are $10.5_{5} \pm 0.29,2.29 \pm 0.03$ and $(0.48 \pm 0.3) \times 10^{-3} \mathrm{deg}^{-1}$, respectively, where $n$ is the number of bond length $l$ in the main chain. The former two values are calculated by ignoring the temperature dependences and the solvent effects, and the last one is calculated by ignoring the solvent effects. No special solvent effect is observed for poly( $p$-chlorostyrene). $\sigma$ values in $\theta$ solvent series with UCST and those with LCST are closely connected with the different dependences of $r_{\mathrm{A}}{ }^{\theta}$ which means the solvent power as mentioned in the text. The temperature coefficient $\mathrm{d} \ln \left\langle\boldsymbol{r}_{0}^{2}\right\rangle / \mathrm{d} T$ of homologous series also depends on that of $r_{\mathrm{A}}{ }^{\theta}$ value.

KEY WORDS Poly( $p$-Chlorostyrene) / Theta Solvent / LCST /

UCST / Viscosity Plot / Unperturbed Chain Dimension / Temperature Coefficient / Solvent Effect /
\end{abstract}

Recently, some authors ${ }^{2-7}$ have carried out investigations on the unperturbed dimension, its temperature coefficient and the solvent effect in polystyrene by measuring the intrinsic viscosities in homologous theta solvents and stress-temperature at constant pressure. These experimental results, especially, those of Orofino, et al., ${ }^{4,7}$ which have been considered to be the most reliable experimental values, were interpreted with the calculations based on the interdependent-rotational model for a chain conformation given by Flory, et al. ${ }^{8}$ and Abe. ${ }^{9}$ The agreement between experiment and calculation ${ }^{9}$ is reasonable except for the sign of the temperature coefficient.

On the other hand, some authors ${ }^{13-22}$ have investigated the solution properties of halogen derivatives of polystyrene to examine the effects of polar groups introduced in the side group of polystyrene. One of them, Matsumura ${ }^{21}$ reported that there was a considerable solvent effect in poly(o-chlorostyrene) (POCS) - methyl ethyl ke- tone system $\left(\theta_{\mathrm{L}}=25^{\circ} \mathrm{C}\right.$, where $\theta_{\mathrm{L}}$ denotes the theta temperature for the system with a lower critical solution temperature (LCST)) when compared with POCS-toluene system. However, the investigations for the systems of halogen derivative of polystyrene with LCST have been seldom made both experimentally and theoretically. In this paper, the experimental data of poly ( $p$-chlorostyrene) (PPCS) as halogen derivative of polystyrene are offered as the first step.

To obtain the information on the unperturbed dimension, two procedures have been used: one is based on the theoretical equations between the molecular weights and intrinsic viscosities in good solvent systems, and the other is based on the direct measurements of intrinsic viscosities in theta solvent systems.

The former was used by some authors ${ }^{13-16,19,20}$ for PPCS and led to a characteristic ratio of PPCS somewhat smaller than those of polystyrene and poly ( $p$-methylstyrene) ${ }^{15,25}$ With regard to 


\section{Y. IzUMI and Y. MIYAKE}

the latter procedure, the only data so far are those of Kubo, et al. ${ }^{18}$ who have used carbon tetrachloride as theta solvent and those of Matsumura, et al. ${ }^{22}$ who used mixed $\theta$ solvents. The $\theta$ solvents in this work were those discovered recently, as reported in the author's previous paper. $^{23}$

The purpose of this paper is to investigate the unperturbed dimension, its temperature dependence and the solvent effect using several homologous theta solvents that may show similar solvent effects for PPCS. Some fractions of PPCS were measured for the eleven theta solvents. The results are compared with the published data of polystyrene and its halogen derivatives.

\section{EXPERIMENTAL}

\section{Materials}

The synthesis of $p$-chlorostyrene and the preparation of PPCS are the same as in the previous paper. ${ }^{23}$ Two types of PPCS were prepared and fractionated: one was type A employed in the previous paper $^{23}$ and the other was type B prepared at the same conditions except polymerization temperature (i.e., $60^{\circ} \mathrm{C}$ ). Seven fractions in type $A$ and twelve fractions in type $B$ were used for measurements.

The solvents employed for measurements were

Table I. Light-scattering and viscosity data for PPCS at $30^{\circ} \mathrm{Ca}$

\begin{tabular}{ccccccc}
\hline \multirow{2}{*}{$\begin{array}{c}\text { Fractions } \\
\text { (type B) }\end{array}$} & \multicolumn{3}{c}{ Ethylbenzene } & & \multicolumn{2}{c}{ Toluene } \\
\cline { 2 - 3 } \cline { 6 - 7 } & $M_{w} \times 10^{-4}$ & {$[\eta]$} & $k^{\prime}$ & & {$[\eta]$} & $k^{\prime}$ \\
\hline BPF 1 & 125 & 1.018 & 0.46 & & 1.215 & 0.44 \\
2 & 92.3 & 0.821 & 0.52 & & 0.965 & 0.46 \\
3 & 72.1 & 0.750 & 0.49 & & 0.865 & 0.46 \\
4 & 61.6 & 0.668 & 0.50 & & 0.785 & 0.43 \\
5 & $49.7^{\mathrm{b}}$ & - & - & & 0.666 & 0.42 \\
$2-1$ & $49.0^{\mathrm{b}}$ & - & - & & 0.647 & 0.41 \\
$2-2$ & 26.4 & 0.400 & 0.52 & & 0.444 & 0.47 \\
$2-3$ & $20.1^{\mathrm{b}}$ & - & - & & 0.383 & 0.55 \\
2F 1 & 45.3 & 0.526 & 0.51 & & 0.615 & 0.43 \\
1 1F 5 & 63.0 & 0.680 & 0.47 & & 0.790 & 0.46 \\
6 & $35.5^{\mathrm{b}}$ & 0.470 & 0.53 & & - & - \\
\hline \multicolumn{3}{c}{ Average } & 0.50 & & 0.45 \\
\hline
\end{tabular}

${ }^{2} M_{w}$, weight-average molecular weight; [ $\eta$ ], intrinsic viscosity $(\mathrm{d} l / \mathrm{g}) ; k^{\prime}$, Huggins constant.

b Values calculated from eq 1 or 2.

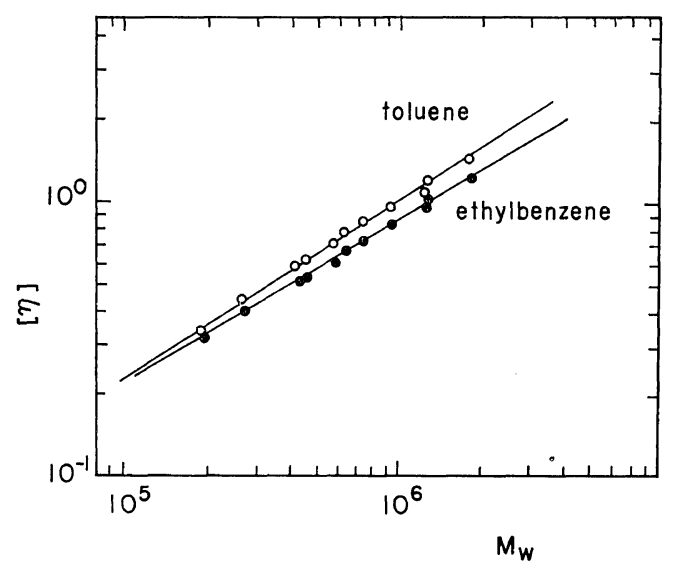

Figure 1. Double logarithmic plots of [ $\eta$ ] against $M_{w}$ for PPCS in ethylbenzene (filled circles) and in toluene (open circles) at $30^{\circ} \mathrm{C}$.

purified by the methods described in the previous paper. $^{23}$

\section{Measurements}

The technical details for light scattering and viscosity measurements have been described in the previous paper. $^{23}$ The results of light scattering and the viscosity measurements for type B are summarized in Table I. The plots of log [ $\eta]$ against $\log M_{w}$ for PPCS in toluene (open circles) and in ethylbenzene (filled circles) at $30^{\circ} \mathrm{C}$ are shown in Figure 1. The straight, full lines are also represented by the equations

$$
\begin{aligned}
& {[\eta]=12.3_{5} \times 10^{-5} M_{w}^{0.65_{3}} \quad \text { in toluene }} \\
& {[\eta]=21.7_{9} \times 10^{-5} M_{w}^{0.60_{1}} \quad \text { in ethylbenzene }}
\end{aligned}
$$

which were given in the previous paper. ${ }^{23}$

The theta temperatures were determined according to the well-known Flory formula. ${ }^{{ }_{\mathrm{a}}}$

\section{RESULTS AND DISCUSSION}

\section{Estimation of Unperturbed Dimensions}

According to the Flory-Fox theory, ${ }^{{ }^{1}}$ the constant $K_{\theta}$ is related to the unperturbed mean square end-to-end distance $\left\langle r_{0}{ }^{2}\right\rangle$ by the equation

$$
K_{\theta}=\Phi\left(\left\langle r_{0}{ }^{2}\right\rangle / M\right)^{3 / 2}
$$

where $M$ is the molecular weight of polymer and $\Phi$ is the universal constant (i.e., $2.87 \times 10^{21}$ is used).

The $K_{\theta}$ values obtained from the Stockmayer- 


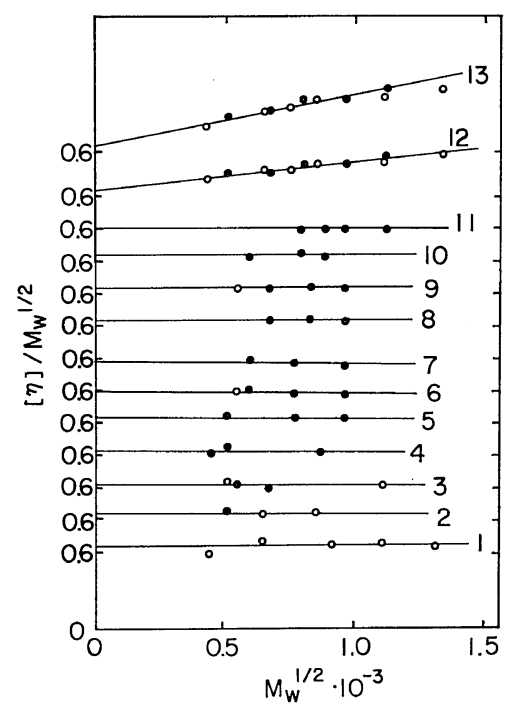

Figure 2. Stockmayer-Fixman plot for PPCS in diluents at each theta temperature except no. (12) and (13): (1) ethylbenzene; (2) isopropylbenzene; (3) carbon tetrachloride; (4) tetrachloroethylene; (5) methyl chloroacetate; (6) ethyl chloroacetate; (7) isopropyl $m$-chloroacetate; (8) isopropyl acetate; (9) $t$-butyl acetate; (10) ethylcarbitol; (11) $n$-butylcarbitol; (12) toluene $\left(30^{\circ} \mathrm{C}\right)$; (13) ethylbenzene $\left(30^{\circ}\right)$. Open circles denote the fractions in type $\mathrm{A}$ and filled circles denote the fractions in type $\mathbf{B}$.
Fixman plot $^{12}$ (S-F plot) at the theta point derived by the phase separation according to Flory's treatment, were assumed to be true values at the theta point. The slopes of the full lines shown in Figure 2 show that the theta points determined are close to the correct values. For the purposes of the present study, a comparison between the $K_{\theta}$ values obtained from the direct measurements using the same fraction of PPCS is better than that from the values obtained from the $\mathrm{S}-\mathrm{F}$ plot, because the latter procedure has a error due to the molecular weight distribution. However, the $\mathrm{S}-\mathrm{F}$ plot procedure is adopted because of a small amount of the same fraction of PPCS.

The $K_{\theta}$ values obtained for eleven theta solvents are summarized in Table II. Without taking into account both the temperature dependence and the solvent effect, an average value of $K_{\theta}=(0.623 \pm$ $0.026) \times 10^{-3}$ is found. It should be noted that the Kurata-Stockmayer plot $^{24}$ of Davis's data leads to $10^{3} K_{\theta}=0.50 \pm 0.03,{ }^{13}$ and also that Kuwahara, et al.,${ }^{15}$ Mohite, et al.,${ }^{19}$ Saito,${ }^{14}$ Noguchi, et al. ${ }^{20}$ and Takamizawa ${ }^{16}$ obtained $10^{3} K_{\theta}=0.52$ $\pm 0.05,0.50,0.567,0.58$, and 0.61 , respectively, from the S-F plot, while Kubo, et al., ${ }^{18}$ and Matsumura, et al., ${ }^{22}$ obtained $10^{3} K_{\theta}=0.544-0.588$ and 0.648 on the basis of the direct measurements, respectively. The present $K_{\theta}$ value is close to the larger values of these. The cause of disagreements

Table II. Molecular parameters of PPCS in theta solvents

\begin{tabular}{|c|c|c|c|c|c|c|c|}
\hline Theta solvents & $\theta,{ }^{\circ} \mathrm{C}$ & $\phi_{1}$ & $r_{\mathrm{A}}^{\theta}$ & $\delta_{\mathrm{s}}{ }^{\theta}$ & $K_{\theta}, \times 10^{3}$ & $\left\langle r_{0}^{2}\right\rangle / n l^{2}$ & $\sigma$ \\
\hline \multicolumn{8}{|l|}{ (Aromatic hydrocarbons) } \\
\hline Ethylbenzene & -14.7 & 0.176 & 2.44 & 9.22 & 0.640 & 10.74 & 2.31 \\
\hline Isopropylbenzene & 59.0 & 0.226 & 3.19 & 8.15 & 0.650 & 10.85 & 2.33 \\
\hline \multicolumn{8}{|l|}{ (Chlorinated hydrocarbons) } \\
\hline Carbon tetrachloride & 50.7 & 0.412 & 4.09 & 8.26 & 0.620 & 10.51 & 2.29 \\
\hline Tetrachloroethylene & 44.4 & 0.546 & 3.39 & 9.07 & 0.620 & 10.51 & 2.29 \\
\hline \multicolumn{8}{|l|}{ (Chlorinated esters) } \\
\hline Methyl chloroacetate & 64.6 & 0.433 & - & $10.2^{\mathrm{a}}$ & 0.640 & 10.74 & 2.31 \\
\hline Ethyl chloroacetate & -1.8 & 0.348 & - & $10.3^{\mathrm{a}}$ & 0.590 & 10.17 & 2.25 \\
\hline Isopropyl chloroacetate & -8.2 & 0.317 & - & $9.7^{\mathrm{a}}$ & 0.565 & 9.88 & 2.22 \\
\hline \multicolumn{8}{|l|}{ (Esters) } \\
\hline Isopropyl acetate & 75.5 & -0.360 & 2.80 & 8.01 & 0.640 & 10.74 & 2.31 \\
\hline$t$-Butyl acetate & 65.4 & -0.323 & 2.75 & $7.74^{\mathrm{a}}$ & 0.640 & 10.74 & 2.31 \\
\hline \multicolumn{8}{|l|}{ (Carbitols) } \\
\hline Ethylcarbitol & 27.8 & -0.696 & 2.66 & 9.6 & 0.645 & 10.80 & 2.32 \\
\hline \multirow[t]{2}{*}{$n$-Butylcarbitol } & 50.1 & -0.489 & 1.85 & 8.6 & 0.605 & 10.34 & 2.27 \\
\hline & & & \multicolumn{2}{|c|}{ Average: } & 0.623 & 10.55 & 2.29 \\
\hline
\end{tabular}

a Values calculated from the method of Small (J. Appl. Chem., 3, 71 (1953)). 
among these data will be discussed in a later section.

The steric factor $\sigma$ defined by

$$
\sigma=\left(\langle r _ { 0 } { } ^ { 2 } \rangle \left\langle\left\langle r_{0}{ }^{2}\right\rangle_{\mathrm{f}}{ }^{1 / 2}\right.\right.
$$

is related to the characteristic ratio by

$$
\left\langle r_{0}{ }^{2}\right\rangle / n l^{2}=(1+\cos \theta) /(1-\cos \theta) \sigma^{2}
$$

where $\left\langle r_{0}{ }^{2}\right\rangle_{\mathrm{f}}$ is the unperturbed dimension for the free-rotational model chain and $\theta$ is the supplement of the skeletal bond angle. For PPCS, $\left\langle r_{0}{ }^{2}\right\rangle \mid n l^{2}=29.2\left\langle r_{0}{ }^{2}\right\rangle / M$ with $n=M / 69.25$ and $l=$ $1.54 \AA$ is used. The steric factor $\sigma$ is also summarized in Table II.

The $\sigma$ value of PPCS, $2.29 \pm 0.03$, is comparable to those of polystyrene $(\sigma=2.22 \pm 0.05)$ and poly( $p$-methylstyrene) $(\sigma=2.17 \sim 2.19) .{ }^{15,25}$ These results may be taken as an indication that the effect of interaction between polar groups at the paraposition on the hindering potential for rotation is not appreciable. In Figure 3, relations of $\sigma$ against the molar volume of side group $V_{\mathrm{X}}$, which serve as a rough measure of the steric hindrance caused by the side groups, are plotted according to Kurata and Stockmayer ${ }^{24}$ and others. ${ }^{24, b-d}$ Polyvinylaromatic series are shown by the upper line which is drawn with the same slope as in Figure 10 of ref $24 \mathrm{c}$ and Figure 11 of ref $24 \mathrm{~d}$. Polyvinyl- $n$-paraffin series are shown by the lower line according to ref $24 \mathrm{~b}$. The lower value of

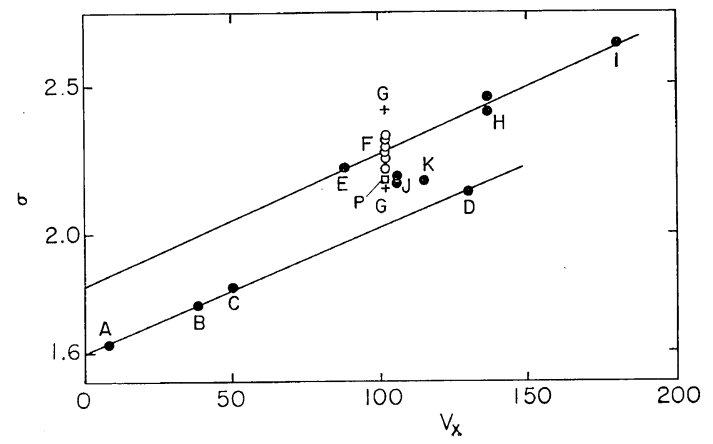

Figure 3. Values of the coefficient of steric hindrance $\sigma$ as function of the molar volume $V_{\mathrm{X}}$ of the side group for different vinyl polymers: A, Polyethylene; $\mathrm{B}$, polypropylene; $\mathrm{C}$, polybutene-1; $\mathrm{D}$, polyoctene-1; $\mathrm{E}$, polystyrene; $\mathrm{F}$ and $\mathrm{P}$, PPCS; G, POCS(poly $(o$-chlorostyrene) $) ; \quad H$, poly(vinylnaphthalene); I, poly(vinylbiphenyl); J, PPMS; K, PDCS. $\sigma$ for a linear chain substituent suggests that they do not introduce as much steric hindrance as a bulky one. $\sigma$ values for PPCS in the present paper are denoted by some open circles across the upper line. The minimum value of the reported data for the same polymer is shown by an open square (denoted by P). $\sigma$ values for POCS $^{21}$ are shown by crossed signs. Figure 3 indicates that PPCS belongs to a poly(vinylaromatic) series rather than a poly(vinyl- $n$-paraffine) series, although this figure shows approximately the shape dependency of substituent for $\sigma$. The results for PPCS and POCS seem to imply that the solvent effect is smaller for PPCS than for POCS, according to Dondos and Benoit. ${ }^{11}$ As, in general, it must be considered that the unperturbed dimension $\left\langle r_{0}^{2}\right\rangle$ in eq 3 depends upon both the temperature and the specific nature of the theta medium, these two effects will be discussed in following sections.

Specific Solvent Effects of Unperturbed Dimensions

Relationships between $K_{\theta}$ values and theta temperatures are shown in Figure 4. Theta solvents with upper critical solution temperature (UCST) are shown by open circles and ones with LCST by filled circles. The $K_{\theta}$ values of PPCSethylbenzene and PPCS - toluene systems obtained from the $\mathrm{S}-\mathrm{F}$ plot at $30.0^{\circ} \mathrm{C}$ are both shown by half filled circles. These values are almost independent of the two procedures used to obtain the $K_{\theta}$ values. Figure 4 denotes that the values of $K_{\theta}$ are not appreciably affected by the solvents except that in ethyl chloroacetate and isopropyl chloroacetate of low $\theta$ temperature.

To examine the solvent effect on $\sigma$ values, $\sigma$

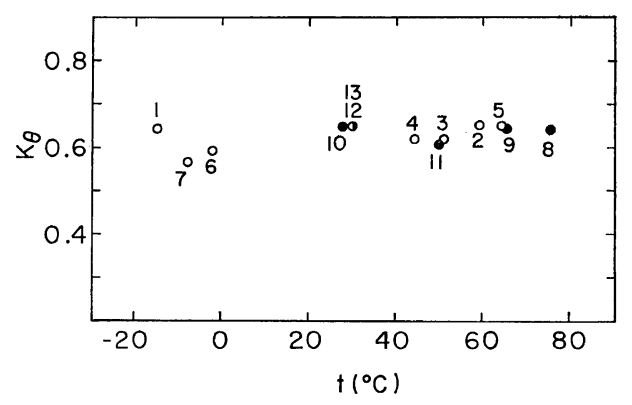

Figure 4. Temperature dependence of $K_{\theta}$ for PPCS: The significance of the numbers is found in Figure 2. 


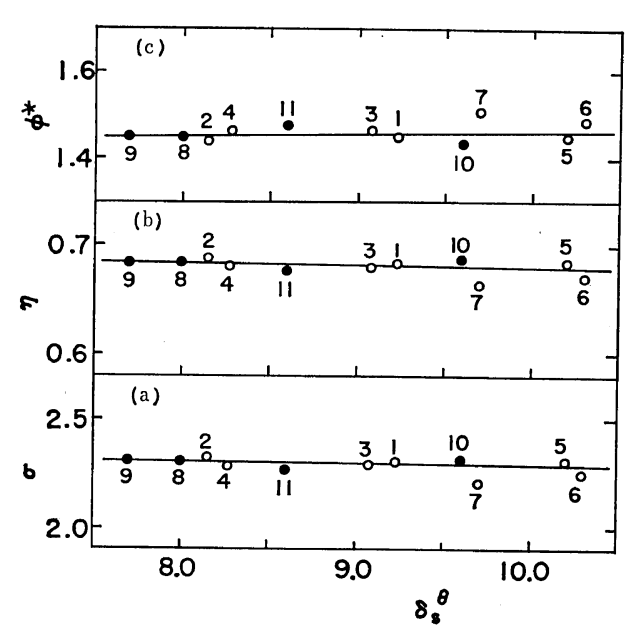

Figure 5. Relationships of (a) $\sigma v s . \delta_{\mathrm{s}}{ }^{\theta}$, (b) $\eta v s$. $\delta_{\mathrm{s}}{ }^{\theta}$ and (c) $\phi^{*} v s . \delta_{\mathrm{s}} \theta$. The significance of numbers is found out in Figure 2.

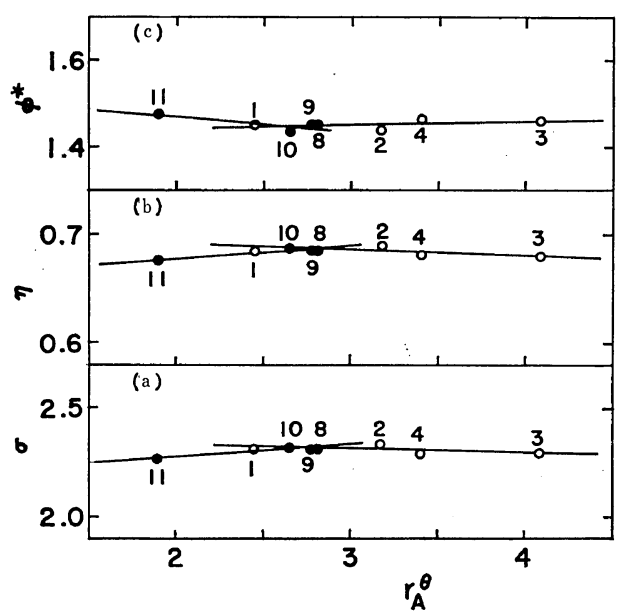

Figure 6. Relationships of (a) steric hindrance $\sigma$ vs. $r_{\mathrm{A}}^{\theta}$, (b) $\eta v s . r_{\mathrm{A}}^{\theta}$ and (c) $\phi^{*} v s . r_{\mathrm{A}}^{\theta}$. The significance of the numbers is found out in Figure 2.

values are plotted against $\delta_{\mathrm{s}}{ }^{\theta}$ values in Figure 5, where $\delta_{\mathrm{s}}{ }^{\theta}$ is the Hildebrand solubility parameter at $\theta$ temperature. Figure 5 shows the similar tendencies in both systems of UCST (denoted by open circles) and LCST (denoted by filled circles), although the $\sigma$ values somewhat scatter about the full line. This situation is also same in plot of $\sigma$ against the molar volume of solvent at $\theta$ temperature $V_{\mathrm{s}}{ }^{\theta}$. As shown in Figure 6, the differences between the series of $\theta$ solvents with UCST and those with LCST have been shown in the plot of $\sigma$ against $r_{\mathrm{A}}{ }^{\theta}$ which denotes the magnitude of solvent power at $\theta$ temperature and whose physical meaning in briefly given in Appendix. This may be explained by the fact that the $r_{\mathrm{A}}{ }^{\theta}$ value reflects the difference between the UCST and LCST systems through the differences in the relative contribution of the three components of solubility parameter. ${ }^{23}$ In order to clarify the relation of $\sigma$ vs. $r_{\mathrm{A}}{ }^{\theta}$, it will be necessary to perform the investigation for a small value of $r_{\mathrm{A}}{ }^{\theta}$, which corresponds to a good solvent, using the $\mathrm{S}-\mathrm{F}$ plot.

For the model chain with fixed bond angles and potentials depending only on the internal rotational angle,

$$
\left\langle r_{0}{ }^{2}\right\rangle / n l^{2}=[(1+\cos \theta) /(1-\cos \theta)][(1+\eta) /(1-\eta)]
$$

is given, where $\eta=\langle\cos \phi\rangle$, and $\phi$ is the internal rotational angle measured from the trans-position. If $\phi$ is allowed to be within a range of $\pm \phi^{*}$, the average of $\cos \phi$ is

$$
\langle\cos \phi\rangle=\sin \phi^{*} / \phi^{*}
$$

Correlations of $\eta v$ s. $r_{\mathrm{A}}{ }^{\theta}$ and $\phi^{*}$ vs. $r_{\mathrm{A}}{ }^{\theta}$ are also shown in Figure 6 and the numerical values are complied in Table III. The similar tendencies as in that of $\sigma$ vs. $r_{\mathrm{A}}{ }^{\theta}$ are seen in these figures, althogh the difference does not appear in the plots of $\eta v s . \delta_{\mathrm{s}}{ }^{\theta}$ and $\phi^{*} v s . \delta_{\mathrm{s}}{ }^{\theta}$ as shown in Figure 5. Figures 5 and 6 , and Table III suggest that the relative energies of rotational isomeric states depend on the solvent to some extent.

Recently, Matsumura reported that $[\eta]_{\theta}$ values for POCS-methyl ethyl ketone and POCStoluene were very different (ca. 33\%). It has

Table III. Internal rotation for PPCS in $\theta$ solvents

\begin{tabular}{lcc}
\hline \multicolumn{1}{c}{ Solvents } & $\eta=\langle\cos \phi\rangle$ & $\phi^{*}(\mathrm{rad})$. \\
\hline Ethylbenzene & 0.684 & 1.451 \\
Isopropylbenzene & 0.689 & 1.438 \\
Carbon tetrachloride & 0.680 & 1.462 \\
Tetrachloroethylene & 0.680 & 1.462 \\
Methyl chloroacetate & 0.684 & 1.451 \\
Ethyl chloroacetate & 0.670 & 1.487 \\
Isopropyl chloroacetate & 0.663 & 1.506 \\
Isopropyl acetate & 0.684 & 1.451 \\
$t$-Butyl acetate & 0.684 & 1.451 \\
Ethylcarbitol & 0.687 & 1.433 \\
$n$-Butylcarbitol & 0.675 & 1.474 \\
\hline
\end{tabular}




\section{Y. IZUMI and Y. MrYAKE}

Table IV. Correlation between temperature coefficient of each solvent series and parameter changes for the polymer-solvent environment

\begin{tabular}{lcccc}
\hline \multicolumn{1}{c}{ Homologous series } & $\mathrm{d} \ln \left\langle r_{0}{ }^{2}\right\rangle / \mathrm{d} T\left(\times 10^{3}\right)$ & $\mathrm{d} \psi_{1} / \mathrm{d} T$ & $\mathrm{~d} \ln r_{\mathrm{A}} \theta / \mathrm{d} T$ & $A=\partial \ln K_{\theta} / \partial \ln r_{\mathrm{A}} \theta$ \\
\hline Average value & $+0.48 \pm 0.3$ & & \\
Aromatic hydrocarbons & $+0.2 \pm 0.3$ & + & + & 0 (nearly zero) \\
Chlorinated hydrocarbons & $0 \quad \pm 0.3$ & - & + & 0 (nearly zero) \\
Chlorinated esters & $+0.86 \pm 0.3$ & + & 0 & 0 (nearly zero) \\
Esters & $0 \quad \pm 0.3$ & - & - & + \\
Carbitols & $-2.5 \pm 0.3$ & + & + \\
\hline
\end{tabular}

a Average value is calculated from the experimental results in spite of each solvent series

been also reported that there are large solvent effects in polysulfone-hexane- $1^{10}$ (ca. $\left.25-30 \%\right)$. To explain the difference between of the specific solvent effects in these polymers, it is suggested to be due to the fact that PPCS has a polar group for from the main chain, while POCS has it near the main chain and polysulfone-hexane-1 has a polar group on the main chain which has great influence on the conformation of the polymer. The solvent effect in PPCS-diluent systems is comparatively small as shown in Figure 4-6. In connection with this point, the remarkable discrepancies between $K_{\theta}$ values of PPCS mentioned above will be due to the differences in the constant $K$ and exponent $\alpha$ in the relation of $[\eta]=K M^{\alpha}$ in spite of using the same method. It may be considered that this reflects a difference in character of the samples employed, i.e., molecular weight distribution, tacticity and so on. However, it will be remained only to point out that the various values of $K_{\theta}$ are reported, ${ }^{13-16,18-20,22}$ because it is not object of this paper to interpret the difference of the values of $K_{\theta}$ in detail.

Temperature Dependence of the Unperturbed Chain

\section{Dimensions}

From eq 3 , temperature coefficient of $\left\langle r_{0}{ }^{2}\right\rangle$ is obtained by

$$
\mathrm{d} \ln \left\langle r_{0}^{2}\right\rangle / \mathrm{d} T=(2 / 3) \mathrm{d} \ln K_{\theta} / \mathrm{d} T
$$

Correlation between the temperature coefficient for each homologous theta solvent and the temperature change of the parameters for the polymer -solvent environment, such as that of entropy parameter $\phi_{1}$ and $r_{\mathrm{A}}{ }^{\theta}$, is complied in Table IV, and the temperature coefficient for each homologous theta solvent is shown in Figure 7. The dependences of $\phi_{1}$ on temperature coefficient in the theta solvents with UCST are consistent with the result of Abe and Fujita, ${ }^{5}$ except for chlorinated hydrocarbons with structures which differ in containing (or not) a double bond. Those in theta solvents with LCST show the reverse tendencies to UCST. The slope of ${r_{A}}^{\theta}$ against $\theta$ in Figure $8 \mathrm{~b}$ suggests that each temperature coefficient is connected more closely with the temperature change of $r_{\mathrm{A}}{ }^{\theta}$ value. Then, eq 8 is rewritten as follows;

$$
\mathrm{d} \ln K_{\theta} / \mathrm{d} T=A \mathrm{~d} \ln r_{\mathrm{A}}{ }^{\theta} / \mathrm{d} T+B
$$

where $A=\left(\partial \ln K_{\theta} / \partial \ln r_{\mathrm{A}}{ }^{\theta}\right)$, and $B=\left(\partial \ln K_{\theta} / \partial T\right)$ may be considered to be nearly zero for all the $\theta$ solvents in the present study. Figure $8 \mathrm{a}$ denotes that the average solvent power coefficient $A$ is a small positive constant for the system with LCST and nearly zero for that with UCST. The sign of the temperature coefficient for each homologous series will be explained by the first term in eq $8^{\prime}$ more quantitatively. The details are shown in Table IV.

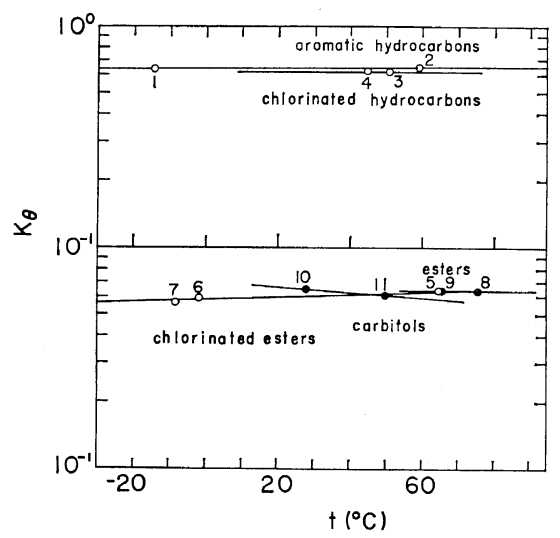

Figure 7. Semilogarithmic plots of $K_{\theta} v s . \theta$ temperatures for PPCS in the homologous theta solvent series. 

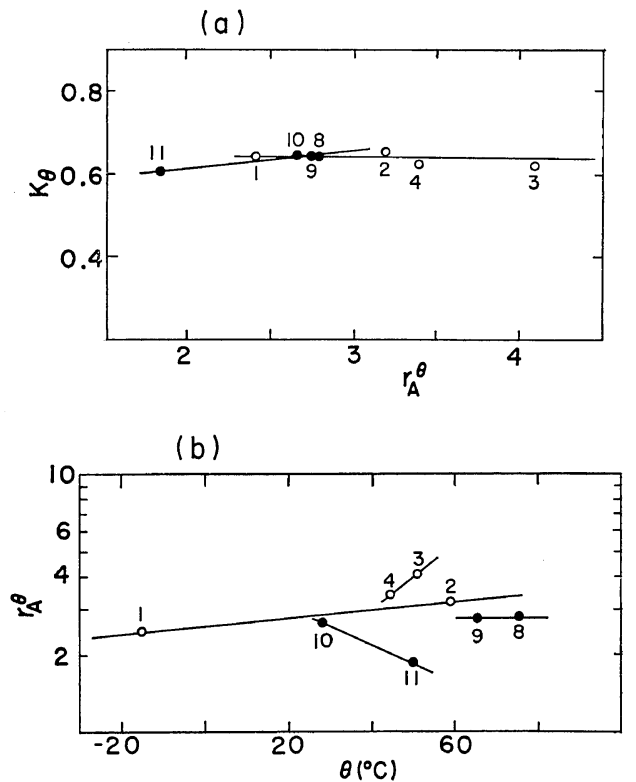

Figure 8. (a) Relationships between $K_{\theta}$ values and parameters $r_{\mathrm{A}} \theta$ vs. $\theta$ temperatures for PPCS in various homologous $\theta$ solvents. The significance of numbers is found out in Figure 2.

The average temperature coefficient is given by

$$
\mathrm{d} \ln \left\langle r_{0}{ }^{2}\right\rangle / \mathrm{d} T=+0.48 \times 10^{-3} \mathrm{deg}^{-1}
$$

irrespective of solvent series used in the present study, as shown in Figure 9. This value is comparable to that of polystyrene obtained by Orofino, et al. ${ }^{4,7}$ However, as seen in Table IV, the values of $\mathrm{d} \ln \left\langle r_{0}{ }^{2}\right\rangle / \mathrm{d} T$ for solvent series of different chemical type differ from each other. This may indicate that the comparison between theoretical and experimental values of $\mathrm{d} \ln \left\langle r_{0}{ }^{2}\right\rangle$ / $\mathrm{d} T^{26}$ is meaningless unless the specific solvent effect is taken into account.

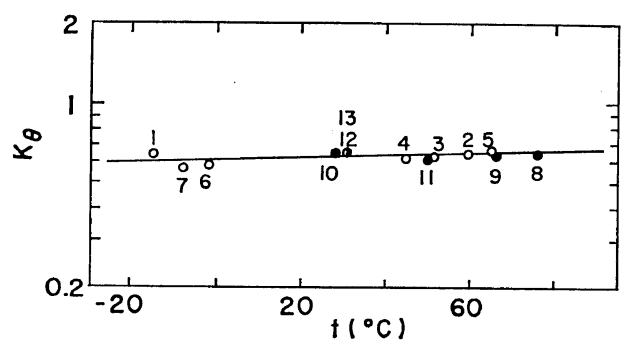

Figure 9. Semilogarithmic plots of $K_{\theta} v s . \theta$ temperatures for PPCS in eleven $\theta$ solvents. The significance of the numbers is found out in Figure 2.

\section{CONCLUSIONS}

The results obtained in this paper were as follows.

(1) The average value of constant $K_{\theta},(0.623 \pm$ $0.026) \times 10^{-3}$, obtained by the direct measurements of intrinsic viscosities in theta solvents, is comparable with that of Takamizawa, ${ }^{16}$ Noguchi, ${ }^{20}$ $\mathrm{Kubo}^{18}$ and Matsumura, ${ }^{22}$ but is somewhat larger than that of Davis, ${ }^{13}$ Kuwahara $^{15}$ and Mohite. ${ }^{19}$ The disagreements among these $K_{\theta}$ values may be ascribed to differences in the molecular weight distribution and the tacticity in the samples employed since the large solvent effect was not found for PPCS and the unperturbed dimension obtained did not depend appreciably on whichever of the two procedures was used to obtained the $K_{\theta}$ values.

(2) The remarkable solvent effect such as for POCS was not observed in spite of the study in both $\theta$ solvents with LCST and UCST. This may be due that PPCS has a polar group far from the main chain. It seems that $\sigma$ values in $\theta$ solvent series with UCST show a different dependence of $r_{\mathrm{A}}{ }^{\theta}$ from that with LCST.

(3) The temperature coefficient has a small positive, negative or zero value depending on the polymer-solvent environment which is denoted by the suitable indices $\phi_{1}$ and $r_{\mathrm{A}}{ }^{\theta}$. Especially, each temperature coefficient seems to be connected more closely with $r_{\mathrm{A}}{ }^{\theta}$ value. A small positive value is obtained for the temperature coefficient, if the average is taken irrespective of solvent series.

Acknowledgement. The authors wish to thank Prof. M. Kaneko for his interest and helpful discussions.

\section{APPENDIX}

$r_{\mathrm{A}}{ }^{\theta}$ denotes the magnitude of solvent power at $\theta$ temperature and is defined by

$$
r_{\mathrm{A}}^{2}=\sum_{j=d, p, h}\left(\delta_{1 j}-\delta_{2 j}\right)^{2}
$$

where suffixes $j=d, p$ and $h$ denote dispersion, polar and hydrogen bonding component, respectively. $\delta_{1 j}$ and $\delta_{2 j}$ denote the $j$ component of the solubility parameter of species 1 and 2, respectively, and are given by

$$
\delta_{i j}^{2}=\Delta E_{i j}^{\vee} / V_{i} \quad(i=1 \text { or } 2)
$$




\section{Y. IzUmI and Y. MIYAKe}

where $\Delta E_{i j}^{\nabla}$ denotes the molar energy of vaporization of component $j$ of species $i$ and $V_{i}$ the molar volume of species $i$. $\quad r_{\mathrm{A}}$ value at any temperature, $r_{\mathrm{A}}{ }^{t}$, is evaluated by taking into account the temperature change of $\Delta E_{i j}^{\nabla}$ and $V_{i}$ in the right-hand side of eq A.2. Details are described in ref 23 .

\section{REFERENCES}

1. P. J. Flory, "Principles of Polymer Chemistry," Cornell Univ. Press, Ithaca, N. Y., 1953, (a) Chapter 13 and (b) Chapter 14.

2. U. Bianchi, J. Polym. Sci., Part A, 2, 3083 (1964); A. Ciferri, ibid., Part A 2, 3089 (1964).

3. T. A. Orofino and J. W. Micky, Jr., J. Chem. Phys., 38, 2512 (1963).

4. T. A. Orofino and A. Ciferri, J. Phys. Chem., 68, 3136 (1964).

5. M. Abe and H. Fujita, ibid., 69, 3263 (1965).

6. U. Bianchi, E. Patrone, and E. Pedemonte, ibid., 70, 3057 (1966).

7. T. A. Orofino, J. Chem. Phys., 45, 4310 (1966).

8. P. J. Flory, J. E. Mark, and A. Abe, J. Amer. Chem. Soc., 88, 639 (1966); see also, P. J. Flory, "Statistical Mechanics of Chain Molecule," Interscience Publishers, New York, N. Y., 1969.

9. A. Abe, Polymer J., 1, 232 (1970).

10. K. J. Ivin, H. A. Ende, and G. Meyerhoff, Polymer, 3, 129 (1962).

11. A.Dondos and H. Benoit, Macromolecules, 4, 279 (1971).

12. W. H. Stockmayer and M. Fixman, J. Polym. Sci., Part C, 1, 137 (1963).
13. J. E. Davis, Ph. D. Thesis, M. I. T. Cambridge, Mass., 1960.

14. T. Saito, Bull. Chem. Soc. Japan, 35, 1580 (1962).

15. N. Kuwahara, K. Ogino, A. Kasai, S. Ueno, and M. Kaneko, J. Polym. Sci., Part A, 3, 985 (1965).

16. K. Takamizawa, Bull. Chem. Soc. Japan, 39, 1186 (1966).

17. K. Kuwahara, K. Ogino, M. Konuma, N. Iida, and M. Kaneko, J. Polym. Sci., Part A, 4, 173 (1966).

18. K. Kubo, K. Ogino, and T. Nakagawa, Nippon Kagaku Zasshi (J. Chem. Soc., Pure Chem. Sect.), 88, 1254 (1967).

19. R. B. Mohite, S. Gundiah, and S. L. Kapur, Makromol. Chem., 116, 280 (1968).

20. Y. Noguchi, A. Aoki, G. Tanaka, and H. Yamakawa, J. Chem. Phys., 52, 2651 (1970).

21. K. Matsumura, Polymer J., 1, 322 (1970).

22. K. Matsumura, M. Fukaya, and K. Mizuno, Bull. Chem. Soc. Japan, 43, 1881 (1970).

23. Y. Izumi and Y. Miyake, Polymer J., 3, 647 (1972).

24. M. Kurata and W. H. Stockmayer, Advan. Polym. Sci., 3, 196 (1963); (b) J. B. Kinsinger and L. E. Ballard, J. Polym. Sci., Part A, 3, (1965); (c) M. Iwata, H. Utiyama, and M. Kurata, J. Macromol. Chem., 1, 701 (1966); (d) L. A. Utracki and R. Simha, Makromol. Chem., 117, 94 (1968).

25. G. Tanaka, S. Imai, and H. Yamakawa, $J$. Chem. Phys., 52, 2640 (1970).

26. J. E. Mark, ibid., 56, 451 (1972). 